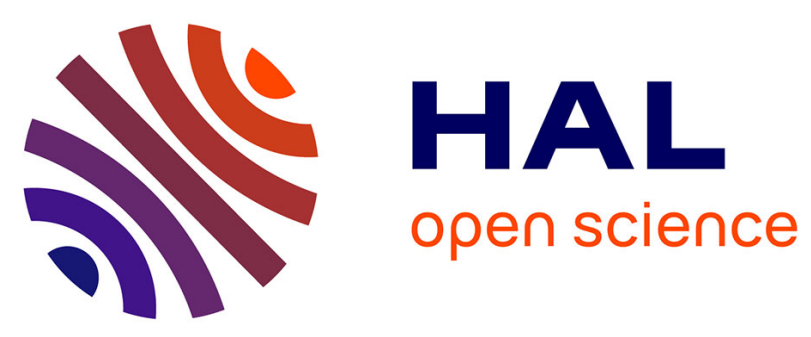

\title{
Does Life resist asynchrony?
}

Nazim A. Fatès

\section{To cite this version:}

Nazim A. Fatès. Does Life resist asynchrony?. Andrew Adamatzky. Game of Life Cellular Automata, Springer London, p. 257-274, 2010, 978-1-84996-217-9. 10.1007/978-1-84996-217-9_14 . inria-00433313

\section{HAL Id: inria-00433313 https://hal.inria.fr/inria-00433313}

Submitted on 18 Nov 2009

HAL is a multi-disciplinary open access archive for the deposit and dissemination of scientific research documents, whether they are published or not. The documents may come from teaching and research institutions in France or abroad, or from public or private research centers.
L'archive ouverte pluridisciplinaire HAL, est destinée au dépôt et à la diffusion de documents scientifiques de niveau recherche, publiés ou non, émanant des établissements d'enseignement et de recherche français ou étrangers, des laboratoires publics ou privés. 


\title{
Does Life resist desynchronisation?
}

\author{
Nazim Fatès ${ }^{1}$ \\ INRIA Nancy Grand-Est - LORIA, Nancy, France. nazim.fates@loria.fr
}

\section{Introduction}

Undoubtedly, Conway's Game of Life - or simply Life - is one of the most amazing inventions in the field of cellular automata. Forty years after its discovery, the model still fascinates researchers as if it were an inexhaustible source of puzzles. One of the most intriguing questions is to determine what makes this rule so particular among the quasi-infinite set of rules one can search. According to W. Poundstone, "Conway wanted to create a game that would be as unpredictable as possible, yet with the simplest possible rules" [18]. Still, what kind of intuition struck Conway when he designed the model? Was it mere chance or is a method for designing a simple rule which yet leads to a surprising behaviour? Is the Life rule somewhat related to real life, and if it so, can we discover models of equal richness by observing natural phenomena?

Our long-term objective is to analyse how the properties of the Life rule are related to the properties of natural systems. In other words, we ask whether Conway's Life resists structural perturbations, where structural means that the perturbations modify the interactions of the components interaction instead of modifying their states. In this paper, our aim is to examine whether the Life rule verifies an important property of living organisms: their robustness to desynchronisation, i.e., their ability to keep a constant behaviour when the updating of their components is modified. We thus study the behaviour of an asynchronous version of Life on a regular and an irregular topology.

To date, these issues haven't received much attention. One reason to explain this lack of interest is that Conway's model is generally considered as a simple metaphor without any serious connection to real life. It is thought of as an example which illustrates how basic local rules can produce a complex and unpredictable behaviour. However, even if the model is metaphor, we want to know if asynchronism allows us to discover novel interesting properties. The next section introduces some bibliographical landmarks to see how this issue has been tackled so far. 


\section{A brief History of the Problem}

The idea to test how Life resists noise dates from as early as 1978 with paper by Schulman and Seiden [20]. They examined how the introduction of a stochastic element in the local evolution rule would perturb the long term evolution of the system. They replaced the deterministic transitions $p_{k, s}-$ which are equal to 0 or 1 if a cell in state $\mathrm{k}$ with $\mathrm{s}$ lives neighbours dies or leaves - by $p_{k, s}(T)$ which are the probabilities to be in state 1 at the next iteration. They take $p_{k, s}(T)=\left(p_{k, s}+d . T\right) /(1+T)$, where $d$ is the current density and $T$ is the stochastic parameter, named "temperature" by analogy with the physical parameter. Interestingly, this parameter is chosen such as not to influence the evolution towards increasing or decreasing the density. The main drawback with this approach is that the definition of the probabilities of transition $p_{k, s}$ is not local: the density should first be computed using the state of the whole grid before the transitions are determined. Nevertheless, the authors analyse the perturbed model with first order and improved mean-field analysis. Their findings can be summarised by saying that: (a) the mean-field analysis fails to predict the evolution of the classical deterministic Life but succeeds with high-temperature models ; (b) more surprisingly, the analysis shows that a phase transition exists which separates two regimes: depending on the value of $T$, the system is either attracted to the null density or to an attractor of density $d^{*}=0.37$. However, a deeper analysis of this phase transition remains yet to be achieved.

Adachi et al. also proposed a modified version of Life. Their model takes into account a "temperature" as a stochastic parameter and uses continuous values for the cells' state [2]. The authors show that there are some stable patterns, such as gliders, which resist a finite amount of noise.

Tackling the question of how Life is affected by asynchronous updating, Bersini and Detours [4] proposed a qualitative study of the model under fully asynchronous updating, i.e., when the updating is sequential and cells are chosen randomly and uniformly without any memory (see [12] for a classification of rules with this updating scheme). The authors observed that Life's behaviour was qualitatively altered by the change of updating. They deduced that asynchrony had a stabilising effect since they could observe the emergence of "labyrinth" patterns that would eventually allow the system to reach a fixed point. However, no systematic study of the phenomenon was conducted, in particular the authors did not examine how this stabilising effect scales with the size of the grid.

The first quantitative study of the change of behaviour was conducted by Blok and Bergersen [5]. They examined Life with the $\alpha$-asynchronous updating, i.e., when each cell has a probability $\alpha$ to be updated at each time step. They identified a continuous change of behaviour of the model depending on $\alpha$. More precisely, they found that Life displays a second-order phase transition which belongs to the directed percolation universality class 
(see below). In this paper, we will proceed to similar experiments but with a different protocol.

Finally, let us mention the stochastic variant of Life which was introduced by Monetti and Albano: they modified the local rule by adding probabilities $p_{\mathrm{s}}$ and $p_{\mathrm{b}}$ for survival and birth, respectively [17]. This change produces a firstorder irreversible phase transition between an "extinct" state where no cell is alive and a "live" state where some structures persist. An improved mean-field analysis was developed to support the experimental observations [16].

We now present formally our model, where, in a first step, the perturbations apply only to the updating procedure (and not to the transition rule). Our starting point is thus similar to the study by Blok and Bergersen, we then extended our scope of analysis to a greater range of conditions.

\section{Asynchronous Life}

Let us consider the square grid $\mathcal{L}=\{1, \ldots, L\} \times\{1, \ldots, L\}$ with periodic boundary conditions (the space is a torus). The state of a cell $c \in \mathcal{L}$ at time $t$ is denoted by $\sigma_{c}^{t} \in\{0,1\}$.

Each cell $c \in \mathcal{L}$ is associated with its neighbourhood $\mathcal{N}(c) \subset \mathcal{L}$. We use the 8-nearest neighbours topology: $\mathcal{N}(c)=\left\{c^{\prime} \in \mathcal{L},\left|c_{x}^{\prime}-c_{x}\right|=1\right.$ or $\left.\left|c_{y}^{\prime}-c_{y}\right|=1\right\}$.

The local rule is the function which gives the next state of a cell according to the state of its neighbours. In the case of Life and its variants, it is defined as an outer-totalistic function, i.e., the application of the local rule on a cell $c$ is a function of only two variables: $\sigma_{c}^{t}$ and $s=\sum_{c^{\prime} \in \mathcal{N}(c)} \sigma_{c^{\prime}}^{t}$. A general expression of the local transition rule of Life is stated with two threshold rules:

- birth rule: a cell in state in state 0 becomes a 1 iff $s \in\left[B_{1}, B_{\mathrm{h}}\right]$;

- survival rule: a cell in state in state 0 remains a 1 iff $s \in\left[S_{\mathrm{l}}, S_{\mathrm{h}}\right]$;

where the thresholds $B_{1}, B_{\mathrm{h}}, S_{\mathrm{l}}, S_{\mathrm{h}}$ are non-zero integers. The original Life is defined with the thresholds $B_{\mathrm{l}}=3, B_{\mathrm{h}}=3, S_{\mathrm{l}}=2$ and $S_{\mathrm{h}}=3$.

Classically, the model isdefined with a synchronous updating, i.e., all cells are updated at each time step. In this paper, we will also consider the asynchronous updating, i.e., only a fraction of cells are updated at each time step. There are various ways of considering asynchronous updating in cellular automata. Here, we will consider two simple schemes:

(a) the $\alpha$-synchronous updating [11]: at each time step, each cell will be updated with probability $\alpha$ or left unchanged with probability $1-\alpha$.

(b) the fully asynchronous updating [12]: at each time step, one cell is chosen uniformly at random and updated, the other cells are left unchanged.

The probability $\alpha$ is called the synchrony rate. When $\alpha=1$ we have the classical synchronous updating, the system is deterministic. For $\alpha<1$, the system becomes stochastic. In order to compare the different simulations, we 
use the rescaled time $\tau$ defined as $\tau=t / \alpha$ for the $\alpha$-asynchronous updating and $\tau=t \cdot n$ for the fully-asynchronous updating. Intuitively, for $\alpha \rightarrow 0$, the $\alpha$-synchronous updating and the fully-asynchronous updating become equivalent under the time rescaling. Indeed, the probability that two neighbouring cells are simultaneously updated becomes negligible, which allow the two processes to be mapped one onto the other given the appropriate transformation. However, note that this is valid only for a bounded rescaled simulation time ; if we consider infinite-time evolutions of the system, the equivalence may not hold any more.

Formally, we model the asynchronism by specifying the set of cells which are updated at each time $t$. This is done by using a function $\Delta: \mathbb{N} \rightarrow \mathcal{P}(\mathcal{L})$. For a fixed updating scheme $\Delta$, the global transition function $F_{\Delta}:\{0,1\}^{\mathcal{L}} \rightarrow$ $\{0,1\}^{\mathcal{L}}$ associates a configuration $\sigma_{c}^{t}$ to its successor $\sigma_{c}^{t+1}$ according to:

$$
\forall t \in \mathbb{N}, \forall c \in \mathcal{L}, \sigma_{c}^{t+1}= \begin{cases}f\left(\sigma_{c}^{t}, s\right) & \text { if } c \in \Delta(t) \\ \sigma_{c}^{t} & \text { otherwise }\end{cases}
$$

where

$$
s=\sum_{c^{\prime} \in \mathcal{N}(c)} \sigma_{c^{\prime}}^{t}
$$

and $f(q, s)$ is the local transition function defined with:

$$
f(0, s)=\left\{\begin{array}{l}
1 \text { if } s=3 \\
0 \text { otherwise }
\end{array} \quad \text { and } \quad f(1, s)=\left\{\begin{array}{l}
1 \text { if } s \in[2,3] \\
0 \text { otherwise }
\end{array} .\right.\right.
$$

Using this formalism, we reformulate our objective as an analysis of the behaviour of global transition rule $F_{\Delta}$ depends on the updating scheme $\Delta$. The word "behaviour" has yet to be defined more precisely ; in this article we will mainly use two functions for quantifying changes of behaviour:

- the density $d(x)$, i.e., the ratio of $1 \mathrm{~s}$ in a configuration $x$.

- the activity $\chi(x)$, i.e., the ratio of unstable cells in a configuration $x$.

These two parameters are only rough projections of the state of the system and clearly, they capture only a small part of what one generally expects by saying "behaviour". However, we will see that they give enough information to observe interesting phenomena, especially when qualitative changes of the behaviour of the system need to be detected.

\section{Assessing Life's Robustness to Desynchronisation}

We now turn our attention to the evaluation of the robustness of the Life rule. We proceed in three steps: (1) an informal observation of the changes induced by asynchrony, (2) a quantification of these changes, (3) an analysis of the phase transition with the tools from statistical physics. 


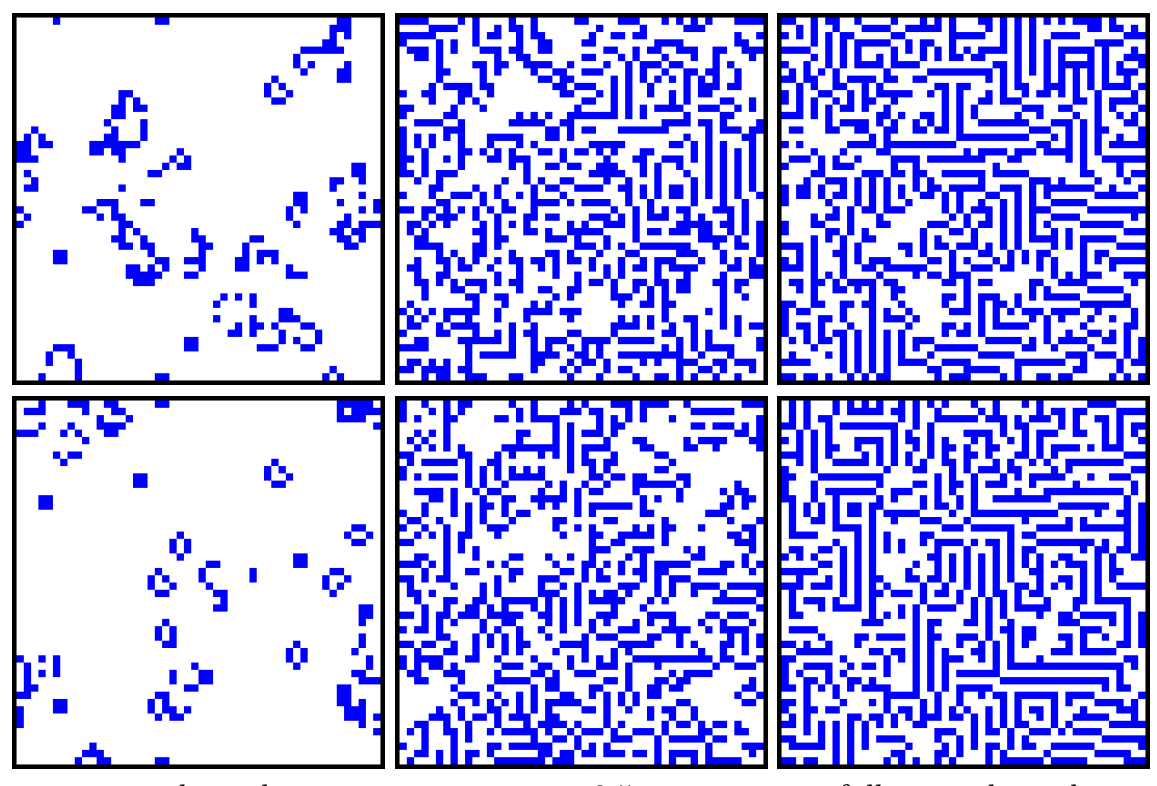

synch. upd.

$\alpha=0.5$

fully-asynch. upd.

Fig. 1. Snapshots of configurations obtained with 3 updating schemes: synchronous (left), $\alpha$-asynchropnous (middle), fully asynchronous (right). Configurations are taken at time $\tau=100$ (top) and $\tau=200$ (bottom).

\subsection{First Experiment}

Figure 1 shows Life's steady state typical configurations for three different updating schemes. It illustrates the influence of the updating on the behaviour of the system: we see that for the asynchronous updating, a new type of pattern has emerged. We call these patterns labyrinths ; their presence on the grid depends on the synchrony rate $\alpha$ : for $\alpha>0.9$, no labyrinth pattern appears, for $\alpha<0.9$, the lower the synchrony rate, the denser the labyrinth patterns. How can we explain this modification?

We repeated the previous experiment by decreasing $\alpha$ by steps of $5 \%$ and we observed that the labyrinth shapes progressively appears when $\alpha<0.9$. For $\alpha<0.8$, the system rapidly reaches a steady state where the density becomes stable. As $\alpha$ decreases, blank spaces in the steady state disappear in favour of the labyrinth shapes; for $\alpha<0.5$, these shapes almost cover the whole grid (see Fig. 1).

\subsection{A Quantification of the Changes}

How does this steady state vary as a function of the synchrony rate? A possible method for quantifying the variation is to measure the average activity of the steady-state. Figure 2 shows the steady state activity $\chi_{\infty}$ as a function of $\alpha$. 
The values are obtained by running a system for a transient of 1000 steps and then averaging the activity of the system for another 1000 steps. It confirms that a qualitative change of behaviour occurs for $\alpha \sim 0.9$.

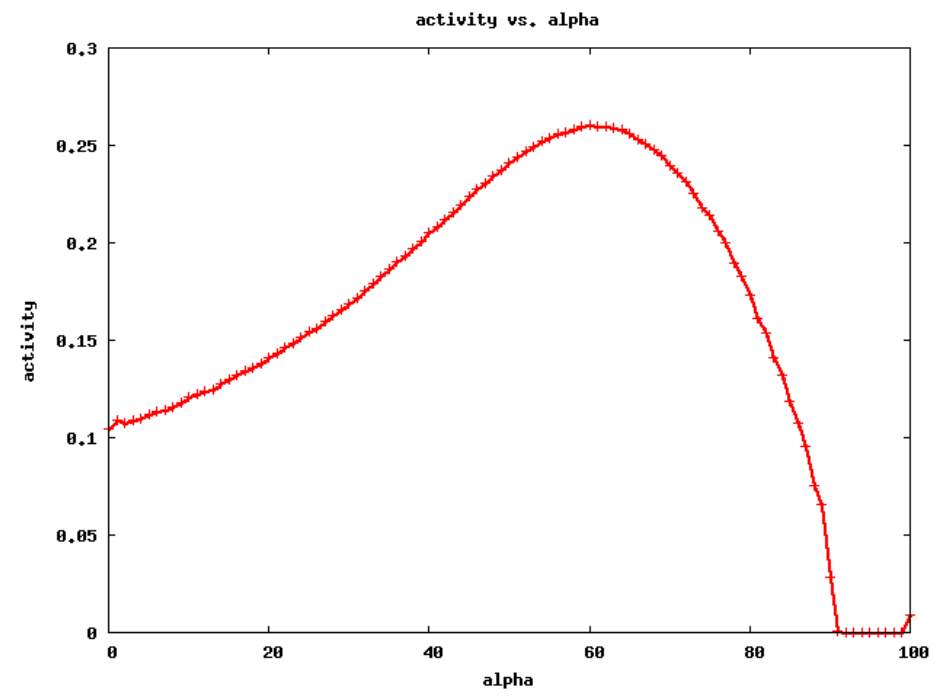

Fig. 2. Steady state activity $\chi_{\infty}$ as a function of the synchrony rate $\alpha$. Grid size is $100 \times 100$. Averages obtained with 12 samples.

As noted by Blok and Bergersen, there also exists a "jump" near $\alpha=1$. This discontinuity is the consequence of a modification of behaviour which occurs as soon as a small amount of noise is introduced: some patterns with a short cycle stability (e.g., the blinkers) are destroyed by the asynchronous updating. Noteworthy is the analogy with similar phenomena observed in $1 \mathrm{D}$ Elementary Cellular Automata (ECA), see [11]. Although for Life, the jump is relatively small (of the order of 0.01), it can be much larger in the case of other cellular automata [10].

If it is easy to explain the change of behaviour near $\alpha=1$, the modification which occurs near $\alpha=0.9$ is rather puzzling at first sight. What kind of transformation the system undergoes at this critical value?

\subsection{A Second-order Phase Transition}

The first quantitative study of the abrupt change of behaviour in the asynchronous Game of Life is due to Blok and Bergersen [6]. The authors showed that the phenomenon was a second-order phase transition: the macroscopic functions that describe the global behaviour as a function of $\alpha$ are continuous but their derivative are discontinuous at the critical point $\alpha_{\mathrm{c}} \sim 0.9$. The 
critical threshold $\alpha_{\mathrm{c}}$ separates two well-distinguished macroscopic behaviours called phases. The first phase $\left(\alpha>\alpha_{\mathrm{c}}\right)$ is the frozen phase, in which the systems evolves with low-density patterns and quickly stabilises to a fixed point. The second phase $\left(\alpha<\alpha_{\mathrm{c}}\right)$ is the labyrinth phase, it is characterised by a steady state with higher density and the absence of a stabilisation on a fixed point (at least for large grid sizes, e.g., for $L>20$ ).

Note that this description is ideal in the sense that it only applies to infinite systems. As simulation imposes to deal with finite systems, how do we then know that we are in presence of a phase transition? This problem is delicate and there exist a great range of methods to answer it. In general, the system is observed near the critical threshold and we measure how a macroscopic description, the order parameter, varies as a function of the lattice size $\mathcal{L}$. Ideally, one should observe that the effect of the lattice size become less and less important, i.e., the order parameter tends to converge to an asymptotic value with regard to the lattice size.

Concomitantly, we should also observe that the order parameter obeys power laws as it gets closer to the threshold. The origin of these power laws comes from the divergence of the system's spatial length scale at the critical point: the system becomes self-similar and its structure follows a fractal pattern. A major discovery in statistical physics is that the power laws that describe the system are not arbitrary: it was observed that very different systems are described by power laws with the same critical exponents. The set of systems which exhibit the same critical exponents is called a universality class. The identification of a universality class is thus a trusted method to show that a brutal change of behaviour is a phase transition (and thus not a mere continuous change in the system's behaviour).

By measuring the value of the steady state activity $\chi_{\infty}$ as a function of the synchrony rate $\alpha$, Blok and Bergersen demonstrated that asynchronous Life's phase transition belonged to the universality class of directed percolation (see [14] for a detailed description). Their protocol consisted in measuring a single critical exponent, i.e., they showed that near criticality, the system obeys:

$$
\chi_{\infty}(\alpha) \sim\left(\alpha-\alpha_{\mathrm{c}}\right)^{\beta}
$$

with $\beta$ being predicted by the directed percolation theory. Although this allowed them to locate the critical threshold at $\alpha_{\mathrm{c}}=0.9083$, it is well-known in the literature that this method alone does not give precise results [14]. Indeed, the closer we get to the critical threshold, the longer we need to wait for the system to stabilise to a steady state. This phenomenon, known as the critical slowing down, is difficult to counterbalance. It often introduces systematic biases in the measures, which can not be "seen" by merely computing the uncertainties in the statistical data. In order to avoid the biases of this method, we follow another protocol which was for instance used by Grassberger for linear stochastic cellular automata [13]. 
The protocol consists of an interactive process: (a) we fix $\alpha$ and start with a random configuration, (b) we monitor the evolution of the order parameter for a long simulation time until we observe a sub-critical or super-critical behaviour ; (c) we repeat the experiment with a value closer to the critical point until we are not able to observe the difference between sub- and supercritical behaviour.

As we expect the evolution of the order parameter to be a power law near criticality, its evolution should thus appear as straight line on a log-log plot. The value of threshold is not known advance, the values we test are thus either sub-critical or super-critical. The critical threshold is approximated by observing the changes of convexity in the plots displayed in log-log scale: a plot with a positive or negative curvature corresponds to a super-critical or a sub-critical behaviour, respectively.

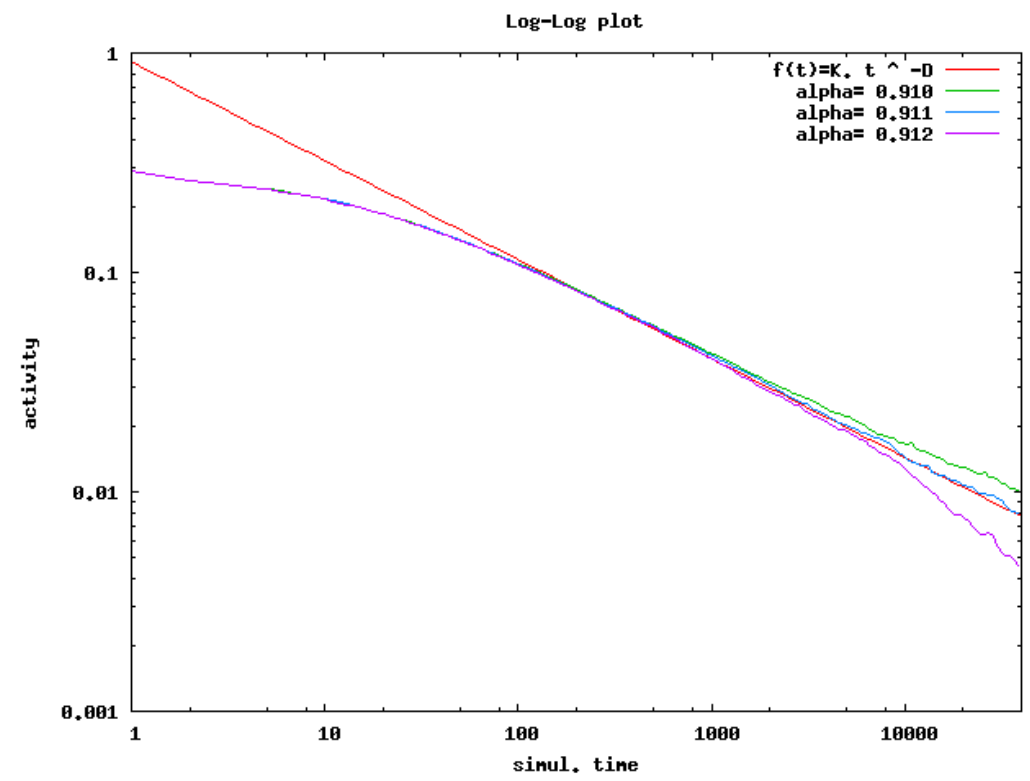

Fig. 3. Evolution of the activity $\chi$ as a function of time ; grid size is $800 \times 800$, averages are taken with 50 samples. the straight line has slope $-\delta=-0.451$.

We tested both the density and the activity as an order parameter and observed that the activity gave much better results. Figure 3 displays different evolutions of the steady state activity $\chi$ for different synchrony rates. We find that for $\alpha=0.912$, the curve bends downwards, which indicates an subcritical regime ("frozen phase"); while for $\alpha=0.910$, the curve bends upward, which indicates a super-critical regime ("labyrinth phase"). We thus locate the phase transition at the point $\alpha_{\mathrm{c}}=0.911 \pm 10^{-3}$. Note that this value, 
although close to value given by Blok and Bergersen is not in agreement with their uncertainty range. As said before, we believe that the small discrepancy comes from the biases which exist in the method they have used. Other experiments are now needed in order to settle this value with greater precision. This could be done for example by measuring the dynamical exponents, starting from an initial condition close to the absorbing phase. In any case, for the critical synchrony rate $\alpha_{\mathrm{c}} \sim 0.911$, we observe a good agreement between the measured slope and the expected value $\delta=0.451$ which corresponds to the directed percolation critical exponent.

\section{Influence of the Initial Condition on the Asymptotic Behaviour}

So far we used only uniform random initial conditions, i.e., configurations where each cell had a probability $d_{\text {ini }}=1 / 2$ to be in state 1 . For the great majority of dynamical systems, the choice of the initial condition generally has an effect in the outcome of a trajectory. What about the asynchronous Life? For $\alpha<\alpha_{\mathrm{c}}$, do we always observe the labyrinth phase regardless of the initial condition? To deal with this question, we first observe the system behaviour from an experimental point of view. Part of this behaviour is then explained using a mean-field analysis and another part is explained with a close-up on how the labyrinth phase develops from specific parts of the lattice.

\subsection{Experimental Approach}

Bagnoli et al. were among the first authors to examine in details the importance of the initial condition in the evolution of the Game of Life [3]. For the classical synchronous updating, they detected two sharp transitions in the asymptotic density $d_{\infty}$ as a function of the initial density $d_{\text {ini }}$. Fig. 4 shows $d_{\infty}=f\left(d_{\text {ini }}\right)$ for the synchronous and asynchronous case $(\alpha=0.5)$. The asymptotic density is approximated by taking a $100 \times 100$ grid and measuring the average density during 1000 steps after a transient time of 5000 steps.

The curve obtained for the synchronous case $\alpha=1$ displays two sharp transitions. The first one is for a small $d_{\text {ini }}$ : there exists a threshold which separates the initial conditions which lead to the "extinct" state $(d=0)$ from the initial conditions which lead to the "frozen" state $(d>0)$. Above this threshold, we observe a saturation phenomenon which indicates that the initial density might not have a great importance in the evolution of the system. A symmetrical situation is observed for $d_{\text {ini }}>0.8$ where the system is again lead to the "extinct" state. Now, if we have a look at the asynchronous updating for $\alpha=0.5$, we notice that: (a) the sharp transition for low $d_{\text {ini }}$ is still present, which shows that the labyrinth phase is not always attained, even for $\alpha<\alpha_{\mathrm{c}}$, (b) the sharp transition for high $d_{\text {ini }}$ has disappeared. How can we explain these two phenomena? 


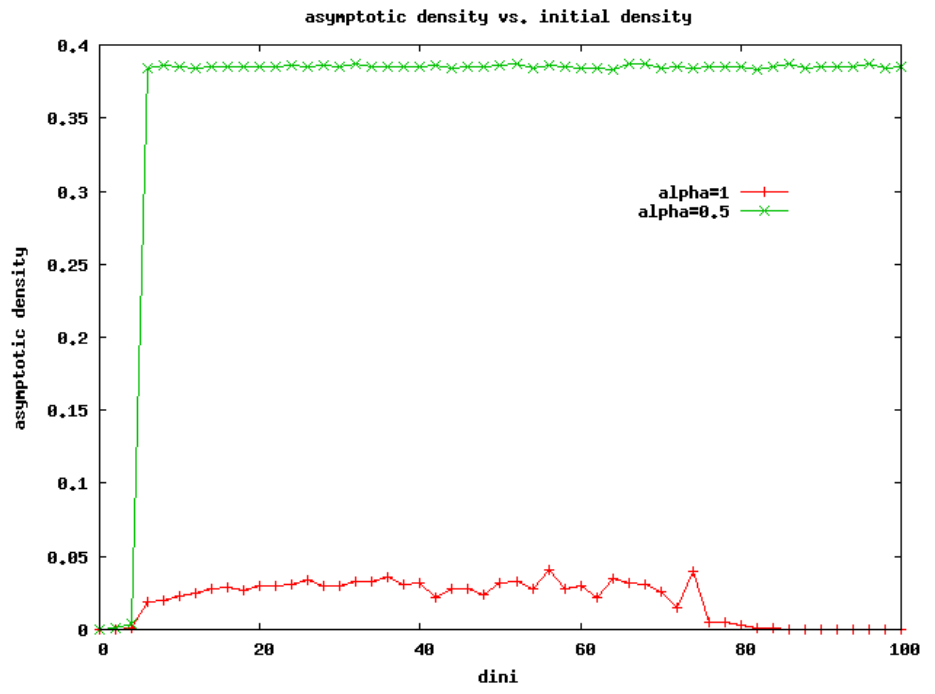

Fig. 4. Asymptotic density as a function of initial density ; grid size is $100 \times 100$.

\subsection{Mean-field Analysis}

Since the evolution of many cellular automata is difficult or impossible to predict analytically, one might need some approximations to make such a mathematical analysis possible. One of the simplest approximations is the mean-field method, which consists in neglecting all spatial correlations. The mean-field analysis is equivalent to considering an infinite system were the cells would be redistributed randomly at each time step.

Let us assume that the density is $d$ at time $t$ and let us calculate the density $d^{\prime}$ at time $t+1$. Since the updating is asynchronous only a fraction $\alpha$ of the cells will be updated. In this subset of updated cells, a cell in state 0 will transform into a 1 (proba. $p_{01}$ ) if and only if it has 3 neighbours (among 8 ) in state 1 . Similarly, a cell that in state 1 will turn into a 0 (probability $p_{10}$ ) if and only if it has not 2 or 3 neighbours. Consequently, we obtain the following set of equations:

$$
d^{\prime}=d+\alpha\left[(1-d) \cdot p_{01}-d \cdot p_{10}\right]
$$

We have

$$
p_{01}=\mathrm{C}_{8}^{3} d^{3}(1-d)^{5}
$$

and

$$
p_{10}=1-\left[\mathrm{C}_{8}^{3} d^{3}(1-d)^{5}+\mathrm{C}_{8}^{2} d^{2}(1-d)^{6}\right]
$$

This results in:

$$
d^{\prime}=d+\alpha d\left[84 d^{2}(1-d)+56 d^{3}(1-d)^{5}-1\right]
$$


We can remark that the solutions points of this equation $\left(d^{\prime}=d\right)$ are the same in the synchronous or asynchronous regime. This implies that the meanfield analysis does not give us any information on how the system is affected by the asynchronous updating! Intuitively, we can observe that if the system is at equilibrium, no matter how many cells are updated since the density of the set of updated cells will not vary. From the mean-field viewpoint, the synchrony rate $\alpha$ acts only as a dilution factor and this why we need to study experimentally the effect of $\alpha$.

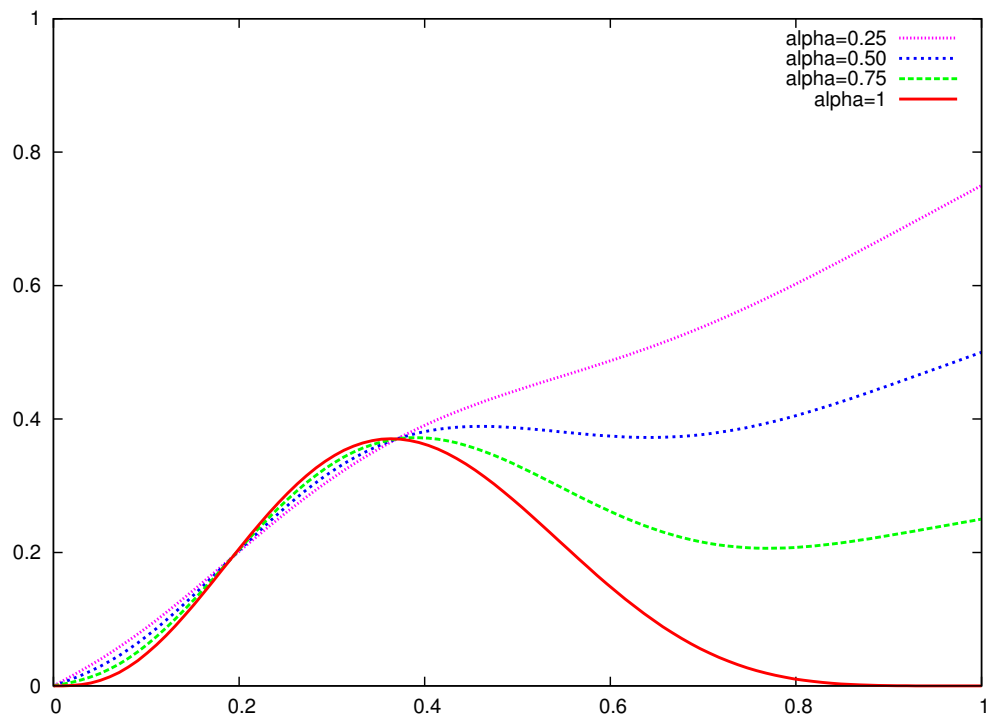

Fig. 5. Mean-field analysis : $d^{\prime}=f(d)$ for different values of $\alpha$.

Figure 5 displays the curve $\mathrm{d}^{\prime}=\mathrm{f}(\mathrm{d})$ for different values of $\alpha$. For $\alpha=1$, we obtain the well-known mean-field analysis with two solutions: $d^{-}=0.19$ is a unstable point while $d^{*}=0.37$ is a stable one. In the limit $\alpha \rightarrow 0$, we simply obtain the identity function; intermediate values result in a "hybrid" function between these two extremal cases. Obviously, the decrease in the synchrony rate mainly affects the evolution of initial configurations with high densities. This observation explains the difference in behaviour between low and high values of $\alpha$ : for a small $\alpha$, only a small fraction of the cells are updated, the density progressively decreases until it reaches the first stable point. By contrast, when $\alpha$ is large, the density goes in one step below the stability limit $d^{*}$ and the system is attracted to the frozen state $d=0$.

Now that we have explained what happens for high densities, let us turn our attention to the other side of the curve. Is there a phase transition for small values of $d_{\text {ini }}$ ? Recall that a necessary condition for having phase transitions is to observe a threshold whose position is independent of the grid size. 


\subsection{A Close-up on Small Initial Densities}

Figure 6 displays the scaling behaviour of $d_{\infty}=f\left(d_{\text {ini }}\right)$ for different grid sizes and for $\alpha=0.5$. The estimation of $d_{\infty}$ is obtained by averaging 200 samples for which we let the system evolve during 1000 time steps as a transient time and then take the average value of the density for another 1000 steps. The curves obtained for the fully-asynchronous updating are similar, although the density saturates at $d_{\max }=0.43$ instead of $d_{\max }=0.38$.

The observations confirm the existence of a strong increase of the asymptotic density $d_{\infty}$ as a function of $d_{\mathrm{ini}}$. However, there is here no clear "signature" of the presence of phase transition as we see no infinite slope appearing for a given threshold value of $d_{\text {ini. }}$. Instead, as the size increases, the slope of the curve also increases but threshold simultaneously seems to be "translated" to the left. Moreover, starting from low- $d_{\text {ini }}$ initial conditions, it is possible to observe that the labyrinth phase develops from very specific parts of the lattice that we call germs, by analogy with crystallography where the germs refers to the elements which can initiate a crystal growth. In order to support our claim that there is no phase transition here, let us analyse how the labyrinth phase develops onto the grid.

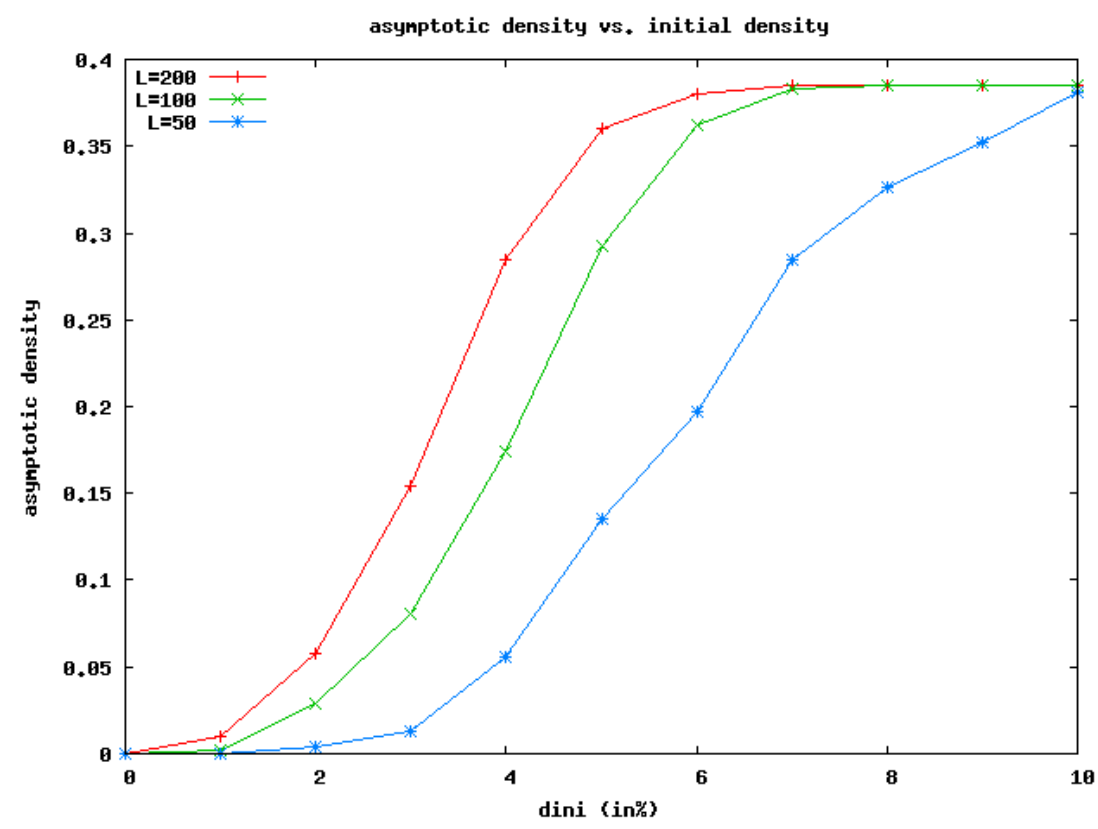

Fig. 6. $d_{\infty}=f\left(d_{\text {ini }}\right)$, close up for small initial densities with $\alpha=0.5$. Averages obtained with 200 samples. 


\subsection{When Germs Colonise the Grid}
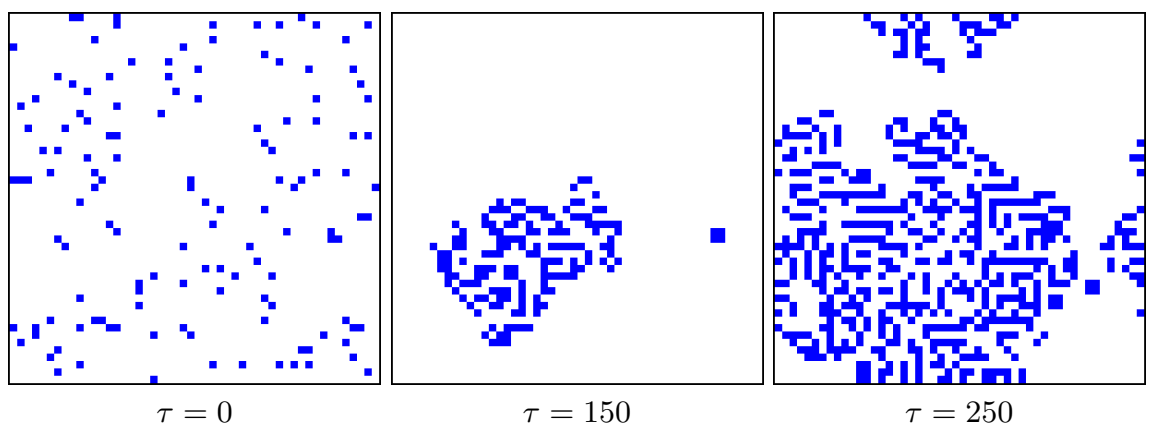

Fig. 7. Colonisation of the grid by a germ. The grid size is $50 \times 50$, the synchrony rate is $\alpha=0.5$, the random configurationw was obtained wityh initial density $d_{\text {ini }}=0.10$.

In a previous paper, we showed that it was possible to predict the shape of the curve $d_{\infty}=f\left(d_{\text {ini }}\right)$ by studying how the germs developed on an empty grid [10]. We considered all $3 \times 3$ germs and examined what was the probability of seeing each germ expand and fill the whole grid, i.e., be the source of the labyrinth phase. The probability of seeing the labyrinth phase was then obtained by grouping the germs according to their number of 1s, and by relating this number to their probability of presence on the grid as a function of the initial density $d_{\text {ini }}$.

We now propose a slightly different method, which is less demanding in simulation time. Instead of studying the $2^{3 * 3}=512$ germs one by one, we will simply set the initial density $d_{\text {ini }}$ and then initialise a $3 \times 3$ block of cells with the central cell set in state 1 and the 8 neighbouring cells initialised randomly with initial density $d_{\text {ini. }}$. All the other cells of the grid are left in state 0 . We then let the system until it either stabilises on a fixed point or it reaches the labyrinth phase. To decide if the labyrinth phase is reached, we use the following criterion: the activity should be higher than the threshold value 0.1 . We verified experimentally that this criterion was fulfilled when the labyrinth phase appeared on the grid. The ratio of labyrinth phases attained over the number of samples gives us $P_{\text {germ }}\left(d_{\text {ini }}\right)$. From this (local) probability, we derive the global probability $P_{\mathrm{LP}}$ to observe the emergence of the labyrinth phase as a function of the initial density $d_{\mathrm{ini}}$. To calculate $P_{\mathrm{LP}}$, we assume total decorrelation between the $3 \times 3$ patterns. The emergence of the labyrinth phase is then obtained if at least one of the germs is the source of the labyrinth phase. As there are $d_{\text {ini }} \cdot L^{2}$ such germs in average, an approximation of the probability of emergence of the labyrinth phase $P_{\mathrm{LP}}$ is given by:

$$
P_{\mathrm{LP}}\left(d_{\mathrm{ini}}\right)=1-\left[1-P_{\text {germ }}\left(d_{\mathrm{ini}}\right)\right]^{d_{\mathrm{ini}} L^{2}}
$$




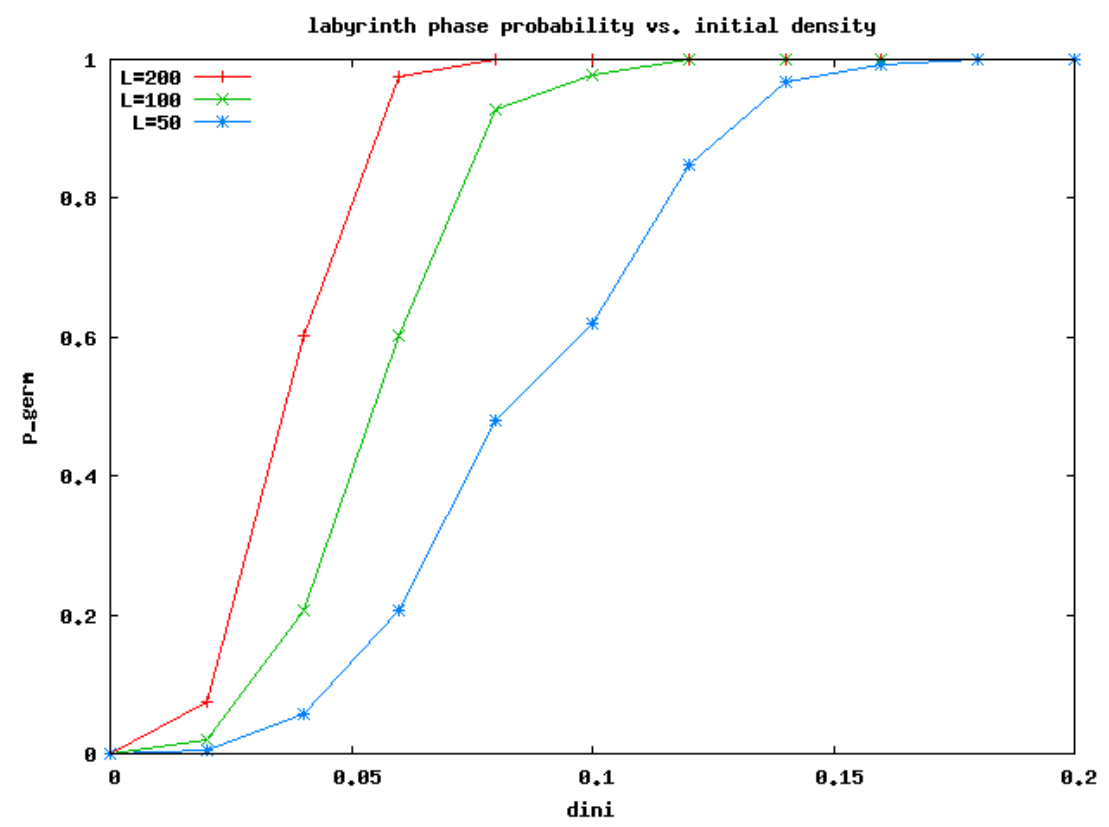

Fig. 8. Estimated probability $P_{\mathrm{LP}}$ to observe the labyrinth phase as a function of the initial density $d_{\text {ini }}$ using the "germ" approximation for $\alpha=0.5$ (see text).

Figure 8 displays $P_{\mathrm{LP}}=f\left(d_{\mathrm{ini}}\right)$ for the fully asynchronous updating scheme and for different lattice sizes. We see that the shape of the curves obtained are similar to the curves of Fig. 6. The curves can be divided into three parts a slow increase for small $d_{\text {ini }}$, followed by a sharp increase for a small range of $d_{\text {ini }}$ and then a saturation for higher values of $d_{\text {ini }}$. As the steady state density reached for $\alpha=0.5$ is close to 0.38 , we observe a qualitative agreement between the plots of Fig. 6 and those of Fig. 8.

This agreement pleads in favour of the non-existence of a phase transition: indeed, if the germ hypothesis is correct, as soon as we have $d_{\text {ini }}>0$, the probability that the labyrinth phase appears and from a germ which "invades" the whole lattice is non-zero, and this probability increases (non-linearly) as a function of the lattice size. These observations can be extended to any $\alpha<\alpha_{c}$ : the probability to observe the labyrinth phase tends to 1 as the grid size tends to infinity. In short, for $\alpha<\alpha_{\mathrm{c}}$, there exists no phase transition with regard to the variation of $d_{\text {ini. }}$. We leave the question open for $\alpha>\alpha_{\mathrm{c}}$, in particular for the synchronous case $\alpha=1$ (the question was raised by Bagnoli et al. [3]). 


\section{Extensions of the Asynchronous Game}

Our examination so far was limited to a Life rule with a desychronised updating but with a regular topology and with its classical definition. In this section, we examine how the behaviour of the asynchronous Life model is affected either by a change of topology or by a change in the thresholds which define the local rule.

\subsection{How important is a regular topology?}

In a similar way that perfect synchrony is not a realistic assumption, it is also possible to question if a perfectly connected grid where each cell has exactly the same number of neighbours is "realistic" (see [8] for a short survey related to this question). In a previous paper, we tackled the question and examined if Life's phase transition was still present if we removed links randomly between a cell and its neighbours. We observed that this operation had a surprising effect: the location of the critical threshold $\alpha_{\mathrm{c}}$ was lowered as the rate of missing links $\epsilon^{-}$was increased. Simultaneously, the phase transition became less and less visible and for $\epsilon \sim 10 \%$, the phase transition was no longer observable [10].

Following the same protocol as described in Sec. 4.3, we performed simulations to test how the phase transition was modified for the missing links perturbation. We verified than the directed percolation was conserved, at least for $\epsilon^{-}<6 \%$. For this range of perturbation, the critical threshold $\alpha_{\mathrm{c}}$ decreases linearly with $\epsilon^{-}$. For a greater amount of perturbations $\left(\epsilon^{-}>6 \%\right)$, we face a "blurring effect", i.e., the phase transition becomes more and more difficult to observe and the critical threshold is hardly located. Similar phenomena were recently observed on a stochastic version of the Greenberg-Hasting cellular automaton [9]. It is an open question to explain how this loss of precision results from the lattice perturbations.

We observed that the phase transition was also perturbed when links were randomly added in a local way, i.e., when some neighbours are counted twice. Similarly, Huang et al. observed that rewiring the links randomly in the lattice (a link has a given probability to be rewired to a cell chosen uniformly in the lattice) produces the same type of phase transition as the asynchronous updating [15]. It is an interesting problem to determine how the non-local topological perturbations and the asynchronous updating combine.

\subsection{How "Frequent" is the Sensibility to Desynchronisation?}

It is now time to go back to our initial question: what is there so special about the Life rule? We saw that it displayed a great sensitivity to its updating scheme and that the evolution of the synchronous and asynchronous systems strongly depended on the initial density. Can this be considered as a "signature" of a complex behaviour? If this is the case, then we would have a 
novel method to search the infinite space of CA rules for Life-like behaviour. We leave the question open to stimulate further research, but before coming to the conclusion of this paper, let us indicate a few facts.

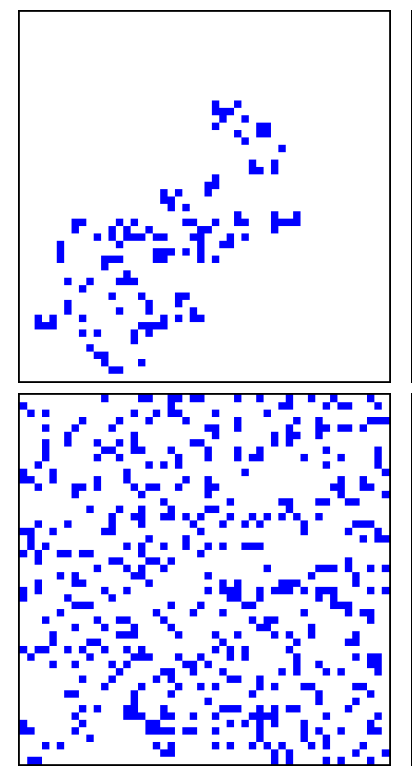

$t=100$

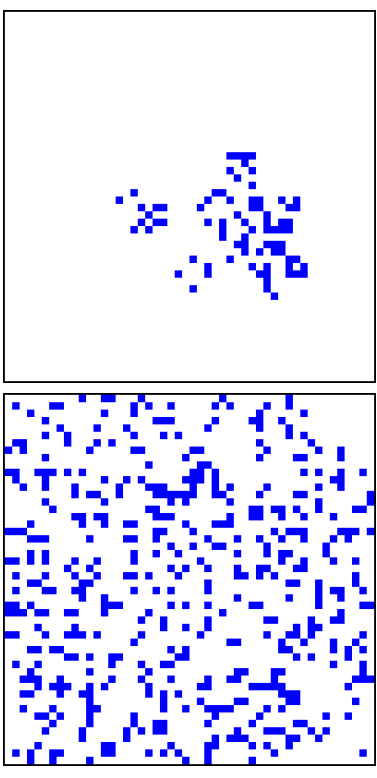

$t=200$

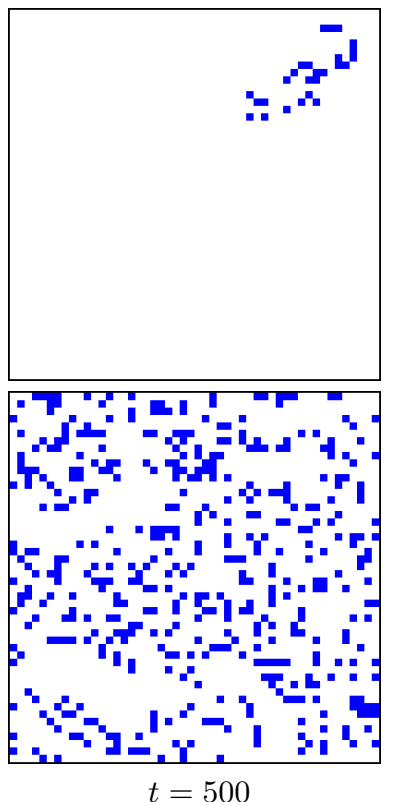

$t=500$

Fig. 9. Three snapshots from the evolution of Kaleidoscope Life : (top) $\alpha=0.90$ critical synchrony rate; (bottom) $\alpha=0.50$ super-critical rate

The Kaleidoscope of Life rule, which was identified by Adachi et al. as being a Turing-universal rule [1] also has a second-order phase transition which is produced by asynchronous updating. The critical threshold appears at $\alpha_{\mathrm{c}} \sim 0.89$ and separates an "extinct" phase were patterns tend to disappear and an "active-sparse" phase with more or less regularly dispersed live sites (see Fig. 9). For this rule, the presence of a phase transition induced by asynchronism raises a challenging question: is it a mere coincidence or is there some possibility of establishing an "equivalence" of behaviour between the two models? (Also note the proximity of the two thresholds.)

To have a larger panel of rules, we briefly explored the larger space of Lifelike rules, where the thresholds are varied. Let us denote by $\mathbb{L}$ abcd the CA rule defined with birth thresholds $B_{\mathrm{l}}=a$ and $B_{\mathrm{h}}=b$ and survival thresholds $S_{1}=c$ and $S_{1}=d$. We found out numerous rules which display asynchronyinduced phase transitions. We indicate for instance the rules: $\mathbb{L} 1756, \mathbb{L} 3643$, $\mathbb{L} 3312, \mathbb{L} 3636, \mathbb{L} 3666$, etc. although there seems to be some "regions" in the rule space where phase transitions are more densely found, we have no clue yet whether there exists a common property shared by these rules. Figure 10 shows 


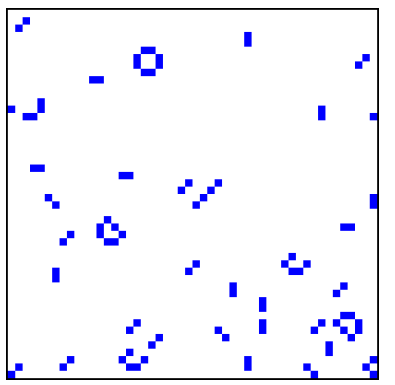

$\mathbb{L} 3312, \alpha=50 \%$

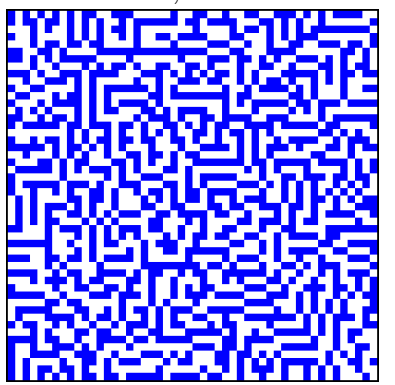

$\mathbb{L} 3666, \alpha=100 \%$

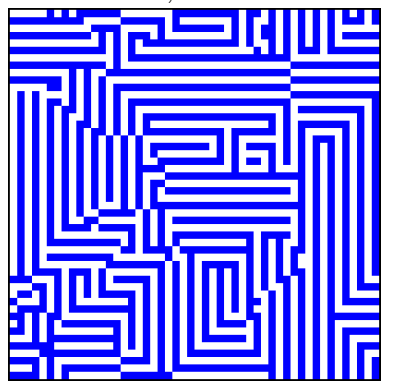

$\mathbb{L} 1756, \alpha=100 \%$

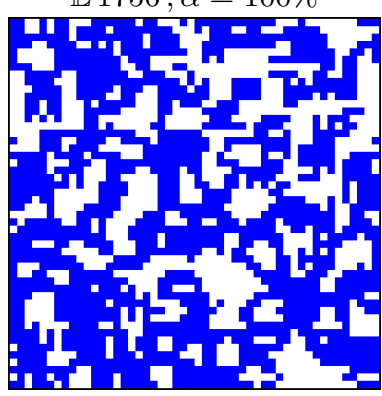

$\mathbb{L} 3636, \alpha=100 \%$

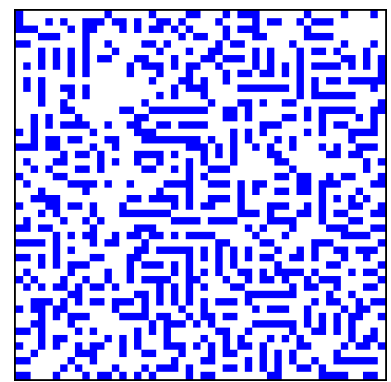

$\mathbb{L} 3312, \alpha=20 \%$

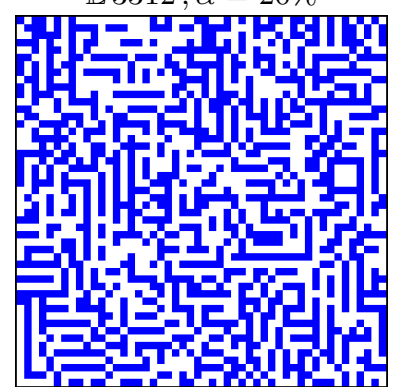

$\mathbb{L} 3666, \alpha=95 \%$

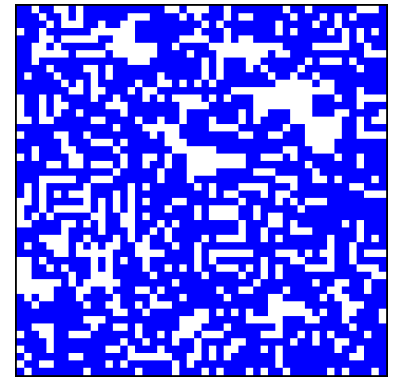

$\mathbb{L} 1756, \alpha=98 \%$

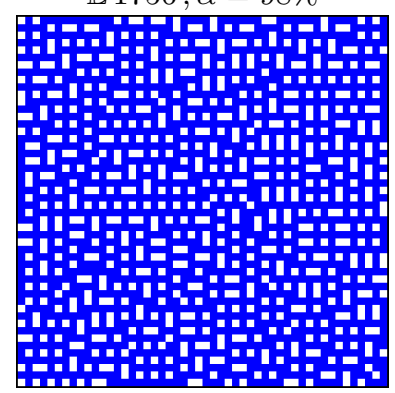

$\mathbb{L} 3636, \alpha=50 \%$
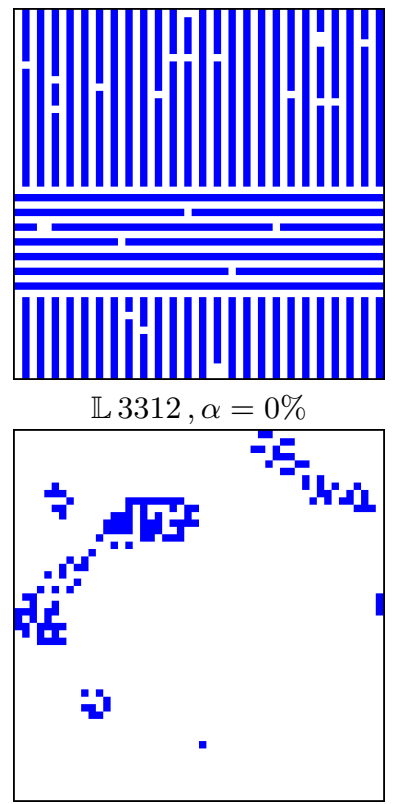

$\mathbb{L} 3666, \alpha=80 \%$

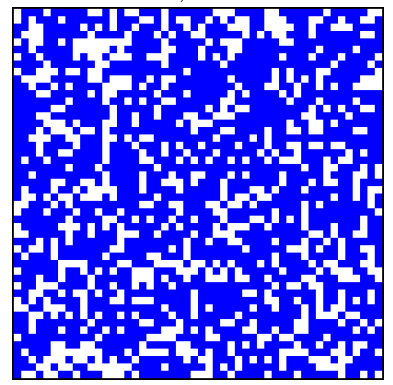

$\mathbb{L} 1756, \alpha=50 \%$

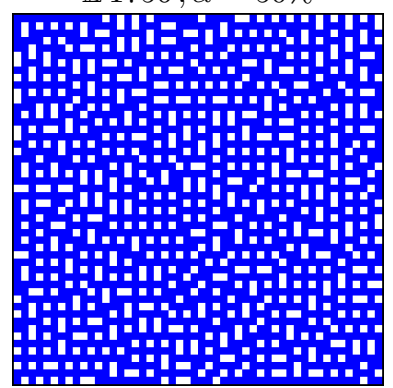

$\mathbb{L} 3636, \alpha=20 \%$

Fig. 10. Snapshots from the steady-state of Extended Life rules. 
different snapshots which display the qualitative changes of behaviour when varying the synchrony rate $\alpha$. We observe that various type of transitions are seen. For $\mathbb{L} 1756$, we even observe two phase transitions: from a "sparse" phase to a "dense-labyrinth" phase and then to an "order-striped" phase. Interested readers may consult the paper by Regnault et al. [19] for similar observations and the study by de la Torre and Mártin [7] for other Extended-Life behaviour in the synchronous case.

\section{Discussion \& Openings}

We studied an asynchronous version of Conway's Game of Life where the cells were updated with partial of full asynchronism. We showed that the variation of the synchrony rate $\alpha$ and initial density $d_{\text {ini }}$ leads to the evolution of the system into qualitatively different steady states, the phases, which we identified as the extinct, the frozen and the labyrinth phases. There exists a critical synchrony rate $\alpha_{\mathrm{c}}$, which separates the frozen phase $\left(\alpha>\alpha_{\mathrm{c}}\right)$ from the labyrinth phase $\left(\alpha<\alpha_{\mathrm{c}}\right)$. This change of behaviour was identified as belonging to the directed percolation universality class, a sign which indicates that the behaviour near the critical point is difficult to predict analytically. Using numerical simulations, the critical threshold $\alpha_{\mathrm{c}}$ was estimated to $\alpha_{\mathrm{c}}=$ 0.911.

We also analysed the dependence of Life on the initial condition and showed that the initial density $d_{\text {ini }}$ was a key factor for predicting the evolution of the system. Here also, for a given lattice size, there exists an abrupt change which depends on the value of $d_{\text {ini }}$ : for $\alpha<\alpha_{\mathrm{c}}$, the emergence of the labyrinth phase is a random process which depends on a complex mechanism that is only partially understood. We proposed to analyse this mechanism by focusing on the "germs", i.e., small "islands" of cells in state 1 in a "sea" of cells in state 0 . The analysis allowed us to understand qualitatively how the labyrinth phase emerges from the germs, but the quantitative predictions have still to be improved. At any rate, these results suggest - by contrast with asynchrony that the abrupt change of behaviour for $d_{\text {ini }}$ is not a phase transition, i.e., there exists no critical initial density that would separate the labyrinth phase from the frozen phase. Finally, we examined how these observations could be generalised to other systems close to the asynchronous Life. As a first step, we indicated that perturbing the topology affected the phase transition by shifting the value of the critical threshold and by making it more difficult to observe (the "blurring effect"). We also provided a list of a few other rules similar to Life where asynchrony also induced phase transitions.

In short, examining the robustness of Life to desynchronisation led us to discover many non-linearities in its behaviour depending on the degree of synchrony and on the initial condition. The mystery of Life is still out there and it is an open question to determine if similar rules can be found in the infinite space of CA rules. 


\section{References}

1. Susumu Adachi, Jia Lee, Ferdinand Peper, and Hiroshi Umeo. Kaleidoscope of life: A 24-neighbourhood outer-totalistic cellular automaton. Physica D, 237(6):800-817, 2008.

2. Susumu Adachi, Ferdinand Peper, and Jia Lee. The Game of Life at finite temperature. Physica D, 198:182-196, 2004.

3. Franco Bagnoli, Raúl Rechtman, and Stefano Ruffo. Some facts of life. Physica A, 171:249-264, 1991.

4. Hugues Bersini and Vincent Detours. Asynchrony induces stability in cellular automata based models. In Rodney A. Brooks and Pattie Maes, editors, 4 th International Workshop on the Synthesis and Simulation of Living Systems ArtificialLifeIV, pages 382-387. MIT Press, 1994.

5. Hendrik J. Blok and Birger Bergersen. Effect of boundary conditions on scaling in the "game of Life". Physical Review E, 55:6249-52, 1997.

6. Hendrik J. Blok and Birger Bergersen. Synchronous versus asynchronous updating in the "game of life". Physical Review E, 59(4):3876-3879, 1999.

7. A. C. de la Torre and H. O. Mártin. A survey of cellular automata like the "game of life". Physica A: Statistical and Theoretical Physics, 240(3-4):560-570, 1997.

8. Nazim Fatès. Critical phenomena in cellular automata: perturbing the update, the transitions, the topology. Technical report, INRIA, 2009.

9. Nazim Fatès and Hugues Berry. Robustness of the critical behaviour in a discrete stochastic reaction-diffusion medium. In Proceedings of the IWNC'09 conference. Springer Japan, 2009. To appear.

10. Nazim Fatès and Michel Morvan. Perturbing the topology of the game of life increases its robustness to asynchrony. In Peter M. A. Sloot, Bastien Chopard, and Alfons G. Hoekstra, editors, Proceedings of the 6th International Conference on Cellular Automata for Research and Industry, volume 3305 of LNCS, pages 111-120. Springer, 2004.

11. Nazim Fatès and Michel Morvan. An experimental study of robustness to asynchronism for elementary cellular automata. Complex Systems, 16:1-27, 2005.

12. Nazim Fatès, Michel Morvan, Nicolas Schabanel, and Eric Thierry. Fully asynchronous behavior of double-quiescent elementary cellular automata. Theoretical Computer Science, 362:1-16, 2006.

13. Peter Grassberger. Synchronization of coupled systems with spatiotemporal chaos. Physical Review E, 59(3):R2520, March 1999.

14. Haye Hinrichsen. Nonequilibrium critical phenomena and phase transitions into absorbing states. Advances in Physics, 49:815-958, 2000.

15. Sheng-You Huang, Xian-Wu Zou, Zhi-Jie Tan, and Zhun-Zhi Jin. Networkinduced nonequilibrium phase transition in the "game of life". Physical Review E, 67:026107, 2003.

16. Roberto A. Monetti. First-order irreversible phase transitions in a nonequilibrium system: Mean-field analysis and simulation results. Physical Review E, 65:016103, 2001.

17. Roberto A. Monetti and Ezequiel V. Albano. Critical edge between frozen extinction and chaotic life. Physical Review E, 52(6):5825, 1995.

18. William Poundstone. The Recursive Universe. William Morrow and Company, New York, 1985. ISBN 0-688-03975-8. 
19. Damien Regnault, Nicolas Schabanel, and Éric Thierry. On the analysis of simple "2d" stochastic cellular automata. In Proceedings of LATA, volume 5196 of $L N C S$, pages 452-463. Springer, 2008.

20. L. S. Schulman and P. E. Seiden. Statistical mechanics of a dynamical system based on conway's game of life. Journal of Statistical Physics, 19:293, 1978. 